\title{
Quantum Coherence of Continuum States in the Valence Band of GaAs Quantum Wells
}

\author{
T. Dekorsy, A. M. T. Kim, G. C. Cho, S. Hunsche, H. J. Bakker, and H. Kurz \\ Institut für Halbleitertechnik, Rheinisch-Westfälische Technische Hochschule Aachen, \\ Sommerfeldstrasse 24, D-52056 Aachen, Germany \\ S. L. Chuang \\ Department of Electrical and Computer Engineering, University of Illinois at Urbana-Champaign, \\ Urbana, Illinois 61801 \\ K. Köhler \\ Fraunhofer Institut für Angewandte Festkörperphysik, D-79108 Freiburg, Germany
}

\begin{abstract}
Quantum beats of heavy and light holes in GaAs quantum wells are investigated in femtosecond time-resolved four-wave mixing and transmission experiments as a function of optical excitation energy. Under nonexcitonic excitation conditions, the four-wave mixing signal disappears due to the immediate loss of the interband coherence of continuum states. In the transmission experiment, the quantum beats are observed up to excess energies of $75 \mathrm{meV}$ above the exciton resonances. The experimental data clearly demonstrate the coherence of continuum states in the valence band. Changes of the beat frequency with the excitation energy are due to the dispersion of the valence bands.
\end{abstract}

Time-resolved investigations of quantum beats in semiconductor quantum wells have received considerable attention in the last few years, since they provide insight into the scattering and coupling mechanisms between light, excitons, free carriers, and phonons in these systems. Most of this work deals with quantum coherence of excitonic states, which may have dephasing rates up to some picoseconds at low lattice temperatures in GaAs based heterostructures. The most commonly used tool for the time-resolved detection of optically excited coherence in semiconductors is four-wave mixing (FWM) spectroscopy [1]. In self-diffracted degenerate FWM, the third order nonlinear interband polarization is detected, which gives a selective sensitivity for the excitonic coherence based on two facts: (i) The signal is proportional to the eighth order of the transition matrix elements [2], which is significantly enhanced at the exciton energy, and (ii) continuum states lose their interband coherence on a time scale of $100 \mathrm{fs}$ resulting in the dominance of the long-living excitonic contribution [3]. The immediate loss of the interband coherence of continuum states arises from the $k$-space band dispersion, which is equivalent to an "inhomogeneous" broadening of interband transitions with the energetic width of the exciting laser spectrum. Furthermore, the excitation of continuum states with excess energy above the band minimum provides additional scattering channels for momentum and energy relaxation so that a decreased interband dephasing time is expected. Several different techniques have been applied for the time-resolved investigation of coherent electronic states in semiconductors, e.g., $\mathrm{THz}$ emission spectroscopy [4-6], time-resolved transmission spectroscopy [2,7], and time-resolved resonant luminescence up-conversion [8]. All these techniques provide distinctly different information on coherent states than FWM. In $\mathrm{THz}$ emission spectroscopy, the detected $\mathrm{THz}$ radiation arises from the real space oscillation of coherent wave packets and is thus not necessarily restricted to excitonic transitions. Therefore, under appropriate excitation conditions, the detected signal may give information on the intraband coherence of the excited states. These observations have been made for the case of nonexcitonic excitation of Bloch oscillations in superlattices [5] and in the $\mathrm{THz}$ emission from free electrons in Landau levels [6]. Specific differences in the intraband and interband dynamics of Bloch oscillations in superlattices could be established more recently [7,9]. All these observations are attributed to the coherence of carriers in the conduction band. Quantum beats involving different hole states have only been observed for excitonic excitation conditions, but no quantum coherence of continuum hole states could be observed [4].

In this Letter, we report on the observation of quantum coherence between continuum states of hole subbands in GaAs quantum wells. For an unambiguous identification of the states involved we measure FWM signals, timeresolved transmission changes (TC), and electro-optic transmission changes (EOTC). We observe quantum beats between heavy and light hole states under nonresonant excitation conditions in both transmission experiments, while the FWM signal decays within the time resolution of our experiment. These experiments demonstrate an unexpectedly slow intraband dephasing of hole states in the continuum of the valence band. 
The sample consists of $40 \mathrm{GaAs}$ quantum wells of $6.5 \mathrm{~nm}$ width separated by $20 \mathrm{~nm} \mathrm{Al}_{0.35} \mathrm{Ga}_{0.65} \mathrm{As}$ barriers. The substrate is removed by wet etching to allow transmission experiments. The $1 s$-heavy hole $(\mathrm{HH})$ and $1 s$-light hole (LH) excitons dominate the cw-absorption spectrum of the sample at $10 \mathrm{~K}$ at 1.593 and $1.614 \mathrm{eV}$, respectively. The experiments are performed with a Kerrlens mode-locked Ti:sapphire laser with a pulse duration of approximately $50 \mathrm{fs}$ and a full width at half maximum (FWHM) of $<40 \mathrm{meV}$ depending on the exact center frequency. The center frequency of the laser is tuned between the HH-LH resonance $(1.6 \mathrm{eV}, \mathrm{FWHM}=$ $39.5 \mathrm{meV})$ and $40 \mathrm{meV}$ above the resonance $(1.64 \mathrm{eV}$, $\mathrm{FWHM}=36 \mathrm{meV}$ ) at $10 \mathrm{~K}$. In the latter case, the laser spectrum is shifted above the excitonic transitions, e.g., the laser intensity at the $\mathrm{HH}$ exciton decreases more than 2 orders of magnitude relative to the excitation with $1.6 \mathrm{eV}$ energy. In order to rule out the possibility that the observed signals may arise from residual excitation of excitonic transitions, further experiments are performed with higher excess energies obtained by increasing the sample temperature. The setup is a conventional pump-probe scheme with a linearly polarized pump beam and a circularly polarized probe beam. FWM is performed in a standard self-diffracted geometry, where the diffracted beam into the direction $2 k_{1}-k_{2}$ is detected with a slow photomultiplier as a function of time delay between the pump and the probe pulse. The TC of the probe intensity are recorded with a photodetector behind the sample. EOTC are detected by introducing a polarizing beam splitter in the probe beam behind the sample and electrically subtracting two polarization components. This signal directly monitors anisotropic transmission changes. The EOTC is free of isotropic contributions resulting from bleaching and broadening of the transitions. For the case of oscillating dipole moments parallel to the growth direction the signal has a contribution due to induced birefringence based on the Pockels effect [10]. Further contributions may arise in resonance with excitonic transitions [11]. All three experiments are performed without changing the excitation conditions. EOTC has a better signal-to-noise ratio than TC since the intensities of one split beam are subtracted from each other, thus giving an effective suppression of intensity fluctuations. A rapid shaker-scanner driven at $100 \mathrm{~Hz}$ is used for modulating the pump-probe time delay to achieve a high sensitivity for all signals. The data are recorded in real time with a $1 \mathrm{MHz}$ analog to digital converter.

In Fig. 1 the TC, EOTC, and FWM signals for resonant $(1.60 \mathrm{eV})$ and off-resonant $(\mathrm{OR}, 1.64 \mathrm{eV})$ excitation are shown. The laser fluence corresponds to an excitation density of $1 \times 10^{10} \mathrm{~cm}^{-2} / 7 \times 10^{9} \mathrm{~cm}^{-2}$ for resonant/OR excitation. For resonant excitation the TC signal is dominated by transmission changes based on Pauli blocking and screening of the excitonic transitions. Under OR excitation these effects decrease by a factor of 4 mainly due to the reduced effects of Pauli blocking and
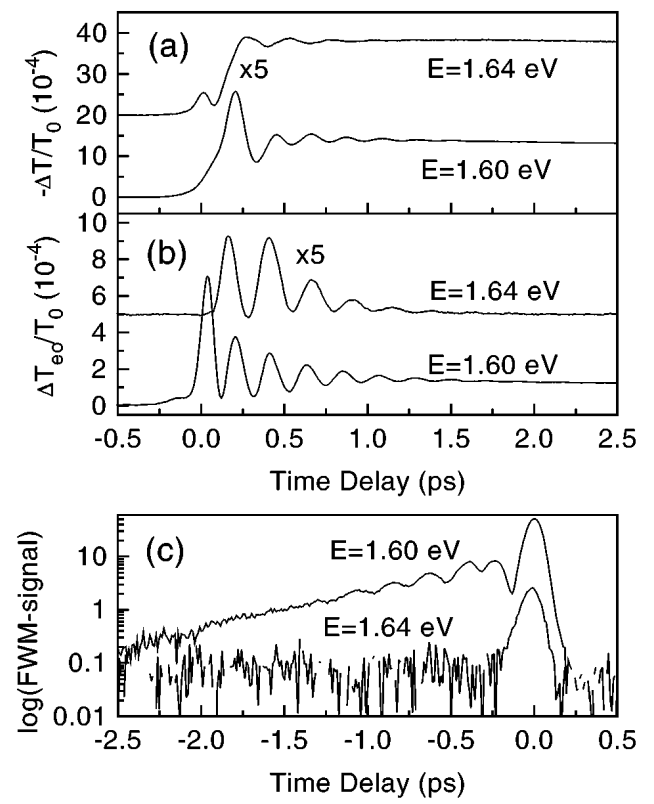

FIG. 1. (a) TC, (b) EOTC, and (c) FWM signals for resonant and off-resonant excitation of the quantum well at $10 \mathrm{~K}$. The curves in (a) and (b) are depicted on a linear vertical scale and the curves for off-resonant excitation are enlarged by a factor of 5. The FWM signal is plotted on a semilogarithmic scale.

broadening for continuum transitions. The TC is modulated by $15 \%$ due to HH-LH beats at resonant excitation and - surprisingly - by $12 \%$ for OR excitation. The HHLH beats are by far more pronounced in the EOTC signal, which is dominated by the oscillatory contribution for resonant and OR excitation. The amplitude of the oscillations decreases by a factor of 4 for OR excitation, which is attributed to resonant contributions to the electro-optic effect in quantum wells [12], resonant anisotropic contributions to a coherent interband polarization [11], and the difference in the excitation density. The dephasing time of the beats decreases by less than a factor of 2 from resonant $(\tau=700 \mathrm{fs})$ to OR excitation $(\tau=360 \mathrm{fs})$. The FWM signal for resonant excitation consists of an initially strong peak due to the excitation of free carriers with a dephasing time shorter than our time resolution. For larger time delays (the time axis is inverted since we detect in the $2 k_{1}-k_{2}$ direction) the signal decays monoexponentially ( $\tau=620 \mathrm{fs}$ ) and is modulated by excitonic HH-LH beats. Under OR excitation, the temporal shape of the signal is symmetric and follows closely the correlation of the laser pulses. The FWM signal amplitude at zero time delay is reduced by 2 orders of magnitude, since only free carrier transitions are excited. The comparison of the data for OR excitation demonstrates phase coherence of free holes in the valence band of the quantum well in the TC and EOTC, while the FWM experiments show that the interband dephasing time of the excited transitions is below the time resolution of the experiment.

In a theoretical treatment on the base of a density matrix formulation it was shown that the FWM intensity and the 
TC signal are given for positive time delays by [2]

$$
\begin{aligned}
I_{\mathrm{FWM}}(\tau) \propto & C\left[\left|\mu_{12}\right|^{4} e^{-2 \gamma_{12} \tau}+\left|\mu_{13}\right|^{4} e^{-2 \gamma_{13} \tau}\right. \\
& \left.+\left|\mu_{12} \mu_{13}\right|^{2} \cos \left(\Delta E_{23} \tau\right) e^{-\left(\gamma_{12}+\gamma_{13}\right) \tau}\right], \\
I_{\mathrm{TC}}(\tau) \propto & \left|\mu_{12} \mu_{13}\right|^{2}\left[1+\cos \left(\Delta E_{23} \tau\right) e^{-\gamma_{23} \tau}\right],
\end{aligned}
$$

respectively. The $\mu_{i, j}$ 's are the transition matrix elements between state $i$ and $j$ and $\tau$ is the time delay. The indices denote the electron, the HH and LH states, respectively. $\gamma_{12}$ and $\gamma_{13}$ denote the interband dephasing rates and $\gamma_{23}$ the intraband dephasing rate. $C$ is a time independent factor containing combinations of the $\mu_{i j}$ 's in the fourth order [2]. The first two terms of the FWM intensity determine the decay of the FWM background modulated by the quantum beats (third term). From the similarities between the TC and the EOTC data we conclude that the latter also provide information on the intraband dephasing rate $\gamma_{23}$.

Under resonant excitation, the observed interband dephasing time of $620 \mathrm{fs}$ as determined from FWM is close to $\gamma_{23}^{-1}$ of $700 \mathrm{fs}$ determined from the dephasing in the transmission experiments [13]. Assuming that intraband dephasing processes also destroy the interband coherence, the interband dephasing has a strong contribution from scattering processes within the valence band. Under OR excitation the interband coherence is destroyed on the time scale of the laser pulses. This is the result of the inhomogeneous broadening introduced by the band dispersion and an increased $k$ space for momentum scattering. Additionally, energy relaxation by LO phonon emission is expected to take place in the conduction band within $170 \mathrm{fs}$ [14]. The decrease of the dephasing time by less than a factor of 2 in the transmission experiments indicates only a small increase of the intraband dephasing processes of continuum states in the valence band under OR excitation.

For higher excess energies the sample temperature is tuned to 100 and $150 \mathrm{~K}$. For excitation with $1.64 \mathrm{eV}$ this corresponds to excess energies above the $\mathrm{HH}$ exciton of $47 \mathrm{meV}(10 \mathrm{~K}), 59 \mathrm{meV}(100 \mathrm{~K})$, and $75 \mathrm{meV}(150 \mathrm{~K})$, respectively. For the highest excess energy the laser intensity at the $\mathrm{HH}$ exciton has decreased to $6 \times 10^{-6}$ of the maximum intensity thus allowing us to entirely exclude excitonic contributions to the observed dynamics. The extracted oscillations obtained from the EOTC for different excess energies are depicted in Fig. 2. The beats are observed for excess energies up to $75 \mathrm{meV}$. Although the amplitude of the beats decreases with increasing excess energies by nearly 2 orders of magnitude, the dephasing time decreases by less than a factor of 2 between resonant and OR excitation at $10 \mathrm{~K}$. For higher excess energies, the dephasing time only slightly decreases. This decrease is mainly due to the intervalence band thermalization of the holes by LO phonon absorption at higher temperatures [15]. Although the total optical excess energies are more than a LO phonon energy, the emission of LO phonons plays a minor role as a scattering mechanism in the valence

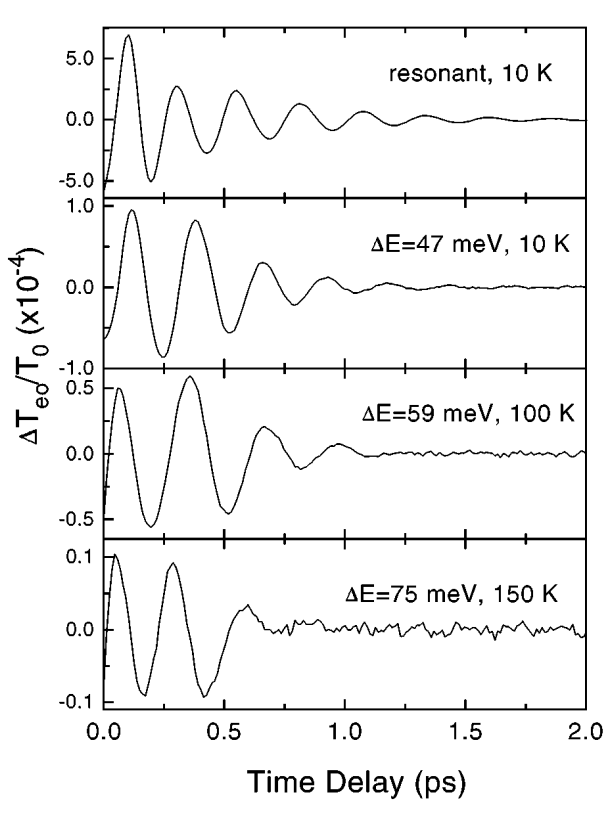

FIG. 2. EOTC for different excess energies as given in the figure.

band, since most of the excess energy is transferred to the conduction band. At $75 \mathrm{meV}$ excess energy the excess energy of electrons is nearly twice the LO phonon energy resulting in a relaxation of the electrons within some $100 \mathrm{fs}$ [14]. The observations in the EOTC are confirmed in the TC, while the FWM signals exhibit only a correlationlike peak at all excess energies.

Differences in the beat frequency are observed for resonant and OR excitation conditions. For a known band dispersion these differences provide information on the momentum parallel to the layers of the continuum states involved in the beating. Figure 3 depicts the Fourier transforms of the EOTC signals of Fig. 2. Under resonant excitation the beat frequency in EOTC, TC, and FWM is the same. The Fourier transform of the EOTC under resonant excitation shows a strong asymmetry that corresponds to a frequency drop from $>6$ to $5 \mathrm{THz}$ in the time domain. This Fano-resonance-like asymmetry is presently of unknown origin and is not observed in FWM and TC. It might be due to differences in the probing process not dominated by excitonic contributions as FWM and TC. For OR excitation at $10 \mathrm{~K}$, the spectrum of the beats is symmetric and shifted by $1 \mathrm{THz}$ to lower frequencies. For higher excess energies, the beat frequency remains about $1 \mathrm{THz}$ below the beat frequency observed under resonant excitation.

For the explanation of the shift of the beat frequencies the dispersion of the valence bands including band mixing has to be taken into account. Figure 4 shows the results of theoretical calculations based on a Luttinger-Kohn Hamiltonian [16]. The conduction band dispersion is assumed to be parabolic. The valence band dispersion 4(a), the transition energies 4(b), and the resulting HH-LH beat frequencies 4(c) are shown. For comparison the 


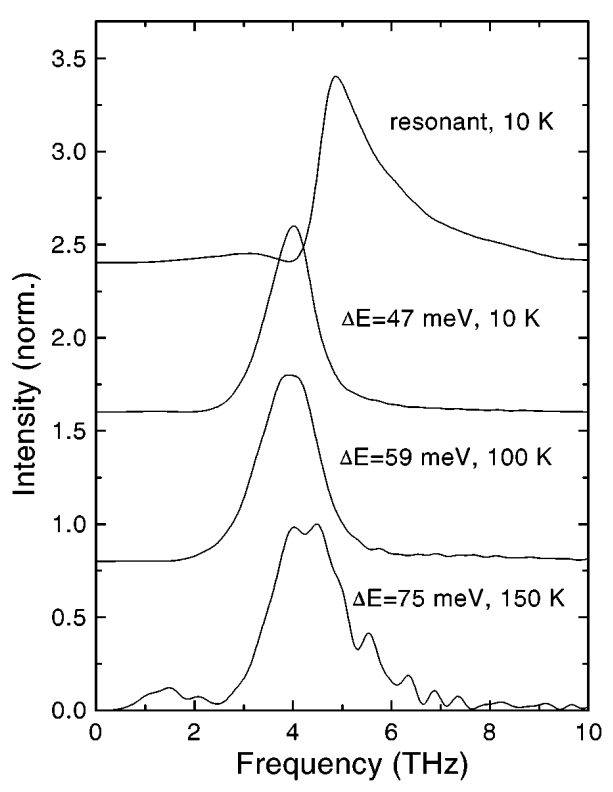

FIG. 3. Fourier transforms of the EOTC shown in Fig. 2.

experimentally determined frequencies are depicted in 4(c) with the momentum for each excess energy derived from 4(b) and the FWHM of the laser pulse. The calculations give a maximum HH-LH splitting for resonant excitation of $21.4 \mathrm{meV}$. A local minimum in the splitting energy

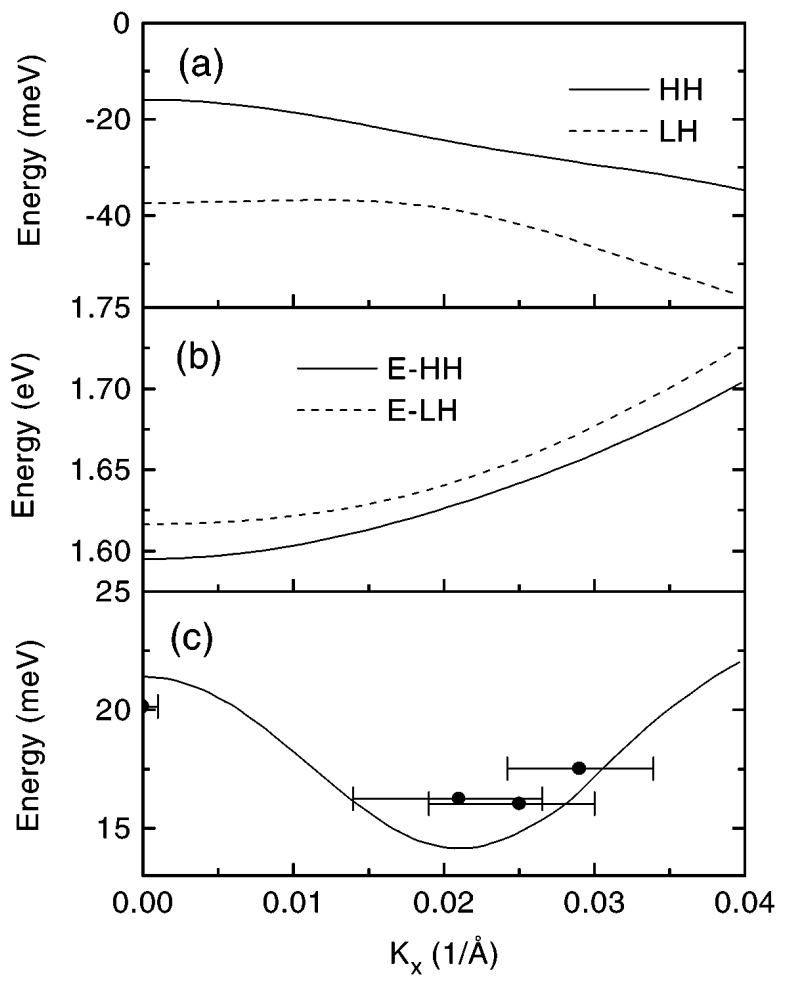

FIG. 4. (a) Calculated valence band dispersion, (b) transition energies, and (c) beat energy versus the in-plane momentum $k_{x}$. The experimental frequencies are also indicated for resonant and off-resonant excitation (circles). The horizontal error bars map the spectral width of the pulses onto the $k_{x}$ momentum. of $14.2 \mathrm{meV}(3.55 \mathrm{THz})$ is observed at $k_{x}=0.02 \AA^{-1}$. The experimental data follow the theoretical curve with an agreement of better than $15 \%$. This agreement provides further evidence that the observed dynamics arise from the coherence of the continuum states in the valence band. The remaining difference is attributed to the strong inhomogeneous energy splitting covered by the broad laser spectrum and the anisotropy of the band dispersion in the $k_{x}-k_{y}$ plane (band warping). This inhomogeneity and the anisotropy, in turn, explain the factor-of-2 increased dephasing for OR excitation. The observed dephasing times are more than an order of magnitude smaller than previously reported hole-spin relaxation times at lower densities [17] and calculated values based on the D'Yakonov-Perel'-like hole scattering mechanism [18]. We therefore attribute the observed dephasing times to hole-hole scattering [19] and to LO-phonon assisted intervalenceband thermalization [15] at elevated temperatures.

In conclusion, we have observed quantum beats of continuum states in the valence band of GaAs quantum wells. The intraband coherence of these states is shown to be longer than the associated interband coherence. This work provides new insight into the fundamental problem of dephasing of continuum states in semiconductors.

We thank P. Haring-Bolivar for useful discussions. This work is supported by the Volkswagen foundation.

[1] B.F. Feuerbacher et al., Solid State Commun. 74, 1279 (1990); J. Kuhl et al., in Coherent Optical Interactions in Semiconductors, edited by R.T. Phillips, NATO Advanced Studies Institute Ser. B, 330 (Plenum, New York, 1994), p. 1, and references therein.

[2] K. Leo et al., IEEE J. Quantum Electron. 28, 2498 (1992).

[3] D.-S. Kim et al., Phys. Rev. Lett. 68, 1006 (1992).

[4] P. C. M. Planken et al., Phys. Rev. Lett. 69, 3800 (1992).

[5] H. G. Roskos et al., Superlattices Microstruct. 15, 281 (1994).

[6] D. Some and A. V. Nurmikko, Phys. Rev. B 50, 5783 (1994).

[7] G. C. Cho et al., Phys. Rev. B (to be published).

[8] H. L. Wang et al., Phys. Rev. Lett. 74, 3065 (1995).

[9] P. Leisching et al., Phys. Rev. B 51, 18015 (1995).

[10] T. Dekorsy et al., Phys. Rev. B 50, 8106 (1994).

[11] R. Lövenich et al. (to be published).

[12] S. H. Kwok et al., Phys. Rev. Lett. 69, 973 (1992).

[13] Both observed dephasing times may be influenced by an additional inhomogeneous broadening of the transitions due to sample inhomogeneities.

[14] C. H. Yang et al., Phys. Rev. Lett. 55, 2359 (1985).

[15] A. M. T. Kim et al., Appl. Phys. Lett. 68, 2956 (1996).

[16] M.S. C. Luo et al., IEEE J. Quantum Electron. 30, 1478 (1994).

[17] P. Roussignol et al., Phys. Rev. B 46, 7292 (1992).

[18] R. Ferreira and G. Bastard, Europhys. Lett. 23, 439 (1993).

[19] M. Woerner and T. Elsaesser, Phys. Rev. B 51, 17490 (1995). 